\title{
A Hydrodynamic Model Including Phase Transition and the Thermal Motion Induced Transverse Momentum Distributions in $p$ - $p$ Collisions at LHC Energies
}

\author{
Zhijin Jiang*, Dongfang Xu, Yu Zhang \\ College of Science, University of Shanghai for Science and Technology, Shanghai, China \\ Email: *jzj265@163.com
}

How to cite this paper: Jiang, Z.J., Xu, D.F. and Zhang, Y. (2018) A Hydrodynamic Model Including Phase Transition and the Thermal Motion Induced Transverse Momentum Distributions in $p$ - $p$ Collisions at LHC Energies. Journal of High Energy Physics, Gravitation and Cosmology, 4, 20-30. https://doi.org/10.4236/jhepgc.2018.41004

Received: October 16, 2017

Accepted: January 9, 2018

Published: January 12, 2018

Copyright $\odot 2018$ by authors and Scientific Research Publishing Inc. This work is licensed under the Creative Commons Attribution International License (CC BY 4.0).

http://creativecommons.org/licenses/by/4.0/

\begin{abstract}
It is widely believed that the matter created in $p$ - $p$ collisions exhibits a similar collective behavior as that formed in heavy ion collisions. In this paper, by taking into account the effects of thermal motion, the transverse momentum distributions of identified charged particles are discussed in the scope of the hydrodynamic model including phase transition. The theoretical model gives a good description to the data collected in $p$ - $p$ collisions at LHC energies for the transverse momentum up to about $p_{T}=1.0 \mathrm{GeV} / c$.
\end{abstract}

\section{Keywords}

Thermal Motion, Relativistic Hydrodynamics, Phase Transition, Transverse Momentum Distribution

\section{Introduction}

In the past decades, a number of bulk observables about charged particles, such as the Fourier coefficients $v_{n}$ of azimuth-angle distributions [1] [2], transverse momentum spectra [3]-[8] and pseudorapidity distributions [9] [10], have experienced a series of extensive investigations in both nucleus and hadron collisions. These investigations have confirmed two facts. One is that the quark-gluon plasma (QGP) might have come into being in nucleus collisions at current RHIC or LHC energies [11] [12] [13] [14] [15]. This also might be true even in hadron collisions at the early lower energies of Intersecting Storage Rings (ISR) and Super Proton Synchrotron (SPS) at CERN [16] [17] [18] [19] [20]. The other is that the matter created in nucleus or hadron collisions shows a clear feature of collective flow, expanding 
nearly like a perfect fluid with very low viscosity [21]-[47].

Apart from collective movement, the quanta of produced matter also have the components of thermal motion. The evolution of the produced matter is the convolution of the collective movement and thermal motion. To clarify the role of thermal motion in the expansions of the produced matter in $p-p$ collisions at LHC energies is the major subject of this paper. To this end, we may as usual ignore the minor collective flow in the transverse directions. The transverse movement of the produced matter is therefore only induced by the thermal motion.

The collective movement of produced matter in the longitudinal direction can be solved analytically. There are a number of schemes in dealing with such precise calculations [21]-[35]. In this paper, the hydrodynamic model proposed by N. Suzuki is employed [27]. Besides the analytical characteristics, the other typical feature of this theoretical model is that it incorporates the effects of phase transition into solutions. This coincides with the current experimental observations as mentioned above. Hence, the employed model is more in line with the realistic situations. In addition, the model is related to the initial temperature of QGP, the sound speed in both partonic and hadronic media, the baryochemical potential, as well as the critical temperature of phase transition. This work may therefore help us understand various transport coefficients of expanding system.

In Section 2, a brief introduction is given to the theoretical model [27], presenting its analytical solutions. The solutions are then used in Section 3 to formulate the invariant multiplicity distributions of charged particles produced in collisions which are in turn compared with the measurements performed by LHC-CMS Collaboration in $p$ - $p$ collisions at LHC energies of $\sqrt{s}=0.9,2.76$ and $7 \mathrm{TeV}$ [3], respectively. The last Section 4 is about conclusions.

\section{A Brief Introduction to the Model}

Here, for the purpose of completion and application, we shall list the key ingredients of the adopted model [27].

1) The movement of fluid meets continuity equation

$$
\frac{\partial T^{\mu v}}{\partial x^{v}}=0, \mu, v=0,1
$$

where $x^{v}=\left(x^{0}, x^{1}\right)=(t, z), t$ is the time and $z$ is the longitudinal coordinate along beam direction. $T^{\mu v}$ is the energy-momentum tensor, which, for a perfect fluid, takes the form

$$
T^{\mu v}=(\varepsilon+p) u^{\mu} u^{v}-p g^{\mu \nu},
$$

where $g^{\mu v}=g_{\mu v}=\operatorname{diag}(1,-1)$ is the metric tensor.

$$
u^{\mu}=\left(u^{0}, u^{1}\right)=\left(\cosh y_{F}, \sinh y_{F}\right), u^{\mu} u_{\mu}=1,
$$

is the 4-velocity of fluid, $y_{F}$ is its rapidity. $\varepsilon$ and $p$ in Equation (2) are the energy density and pressure of fluid, which fit thermodynamic relations 


$$
\varepsilon+p=T \mathrm{~s}, \mathrm{~d} \varepsilon=T \mathrm{~d} s, \mathrm{~d} p=s \mathrm{~d} T,
$$

where $T$ and $s$ are the temperature and entropy density of fluid, respectively. To close Equation (1), another relation, namely the equation of state

$$
\frac{\mathrm{d} p}{\mathrm{~d} \varepsilon}=\frac{s \mathrm{~d} T}{T \mathrm{~d} s}=c_{s}^{2}
$$

is needed, where $C_{s}$ is the sound speed of fluid, which takes different values in QGP and in hadronic phase.

2) Project Equation (1) to the direction of $u_{\mu}$ and the direction perpendicular to $u_{\mu}$, respectively. This leads to equations

$$
\begin{aligned}
& \frac{\partial\left(s u^{v}\right)}{\partial x^{v}}=0, \\
& \frac{\partial\left(T \sinh y_{F}\right)}{\partial t}+\frac{\partial\left(T \cosh y_{F}\right)}{\partial z}=0 .
\end{aligned}
$$

The first formula is the continuity equation for entropy conservation. The second one means the existence of a scalar function $\phi$ satisfying relations

$$
\frac{\partial \phi}{\partial t}=T \cosh y_{F}, \frac{\partial \phi}{\partial z}=-T \sinh y_{F} .
$$

From $\phi$ and Legendre transformation, Khalatnikov potential $\chi$ is introduced via

$$
\chi=\phi-t T \cosh y_{F}+z T \sinh y_{F} .
$$

In terms of $\chi$, the variables $t$ and $z$ can be expressed as

$$
\begin{aligned}
& t=\frac{e^{\theta}}{T_{0}}\left(\frac{\partial \chi}{\partial \theta} \cosh y_{F}+\frac{\partial \chi}{\partial y_{F}} \sinh y_{F}\right), \\
& z=\frac{e^{\theta}}{T_{0}}\left(\frac{\partial \chi}{\partial \theta} \sinh y_{F}+\frac{\partial \chi}{\partial y_{F}} \cosh y_{F}\right),
\end{aligned}
$$

where $T_{0}$ is the initial temperature of fluid and $\theta=\ln \left(T_{0} / T\right)$. Through above equations, the coordinate base of $(t, z)$ is transformed to that of $\left(\theta, y_{F}\right)$, and the first formula of Equation (6) is translated into the so called telegraphy equation

$$
\frac{\partial^{2} \chi}{\partial \theta^{2}}-2 \beta \frac{\partial \chi}{\partial \theta}-\frac{1}{c_{s}^{2}} \frac{\partial^{2} \chi}{\partial y_{F}^{2}}=0, \beta=\frac{1-c_{s}^{2}}{2 c_{s}^{2}} .
$$

3) Along with the expansions of matter created in collisions, it becomes cooler and cooler. As its temperature drops from the initial $T_{0}$ to the critical $T_{c}$, phase transition occurs. The matter transforms from QGP state to hadronic state. The produced hadrons are initially in the violent and frequent collisions. The major part of these collisions is inelastic. Hence, the abundances of identified hadrons are in changing. Furthermore, the mean free paths of these primary hadrons are very short. The movement of them is still like that of a fluid meeting Equation (10) with only difference being the value of $c_{s}$. In QGP, $c_{s}=c_{0}=1 / \sqrt{3}$, which is the sound speed of a massless perfect fluid, being the maximum of $c_{s}$. In the 
hadronic state, $c_{s}=c_{h}<c_{0}$. At the point of phase transition, that is as $T=T_{c}$, $c_{s}$ is discontinuous.

4) The solution of Equation (10) for the sector of QGP is [27]

$$
\begin{aligned}
& \chi_{0}\left(\theta, y_{F}\right)=\frac{q_{0} c_{0}}{2} \mathrm{e}^{\beta_{0} \theta} I_{0}\left(\beta_{0} c_{0} \sqrt{y_{0}^{2}(\theta)-y_{F}^{2}}\right), \\
& \beta_{0}=\frac{1-c_{0}^{2}}{2 c_{0}^{2}}=1, \quad y_{0}(\theta)=\frac{\theta}{c_{0}},
\end{aligned}
$$

where $q_{0}$ is a constant determined by tuning the theoretical results to experimental data. $I_{0}$ is the 0 th order modified Bessel function of the first kind.

In the sector of hadrons, the solution of Equation (10) is [27]

$$
\chi_{h}\left(\theta, y_{F}\right)=\frac{q_{0} c_{0}}{2} B(\theta) I_{0}\left[\lambda\left(\theta, y_{F}\right)\right] \text {, }
$$

where

$$
\begin{aligned}
& B(\theta)=\mathrm{e}^{\beta_{h}\left(\theta-\theta_{c}\right)+\beta_{0} \theta_{c}}, \lambda\left(\theta, y_{F}\right)=\beta_{h} c_{h} \sqrt{y_{h}^{2}(\theta)-y_{F}^{2}}, \\
& \beta_{h}=\frac{1-c_{h}^{2}}{2 c_{h}^{2}}, y_{h}(\theta)=\frac{\theta-\theta_{c}}{c_{h}}+\frac{\theta_{c}}{c_{0}}, \theta_{c}=\ln \left(\frac{T_{0}}{T_{c}}\right) .
\end{aligned}
$$

It is evident that, if $c_{h}=c_{0}$, then $\beta_{h}=\beta_{0}, B(\theta)=\mathrm{e}^{\beta_{0} \theta}, y_{h}=y_{0}$, and thus $\chi_{h}=\chi_{0}$. At the point of phase transition, $T=T_{c}, \theta=\theta_{c}, B(\theta)=\mathrm{e}^{\beta_{0} \theta_{c}}$, $y_{h}=y_{0}=\theta_{c} / c_{0}$. Then

$$
\begin{aligned}
& \left.\chi_{0}\left(\theta, y_{F}\right)\right|_{T=T_{c}}=\frac{q_{0} c_{0}}{2} \mathrm{e}^{\beta_{0} \theta_{c}} I_{0}\left(\beta_{0} \sqrt{\theta_{c}^{2}-c_{0}^{2} y_{F}^{2}}\right), \\
& \left.\chi_{h}\left(\theta, y_{F}\right)\right|_{T=T_{c}}=\frac{q_{0} c_{0}}{2} \mathrm{e}^{\beta_{0} \theta_{c}} I_{0}\left(\frac{\beta_{h} c_{h}}{c_{0}} \sqrt{\theta_{c}^{2}-c_{0}^{2} y_{F}^{2}}\right),
\end{aligned}
$$

$\left.\chi_{h}\left(\theta, y_{F}\right)\right|_{T=T_{C}} \neq\left.\chi_{0}\left(\theta, y_{F}\right)\right|_{T=T_{c}}$. That is, the potential $\chi$ is discontinuous at point of $T=T_{c}$.

\section{The Thermal Motion Induced Transverse Momentum Distributions of Identified Charged Particles}

With Khalatnikov potential $\chi$, the rapidity distributions of fluid read as [26]

$$
\frac{\mathrm{d} N}{\mathrm{~d} y_{F}}=\frac{q_{0} c_{0}}{2} A\left(\cosh y \frac{\mathrm{d} z}{\mathrm{~d} y_{F}}-\sinh y \frac{\mathrm{d} t}{\mathrm{~d} y_{F}}\right),
$$

where $A$ is the cross area of overlap region of collisions. Inserting Equation (9) into above equation, the part in the round brackets becomes

$$
\begin{aligned}
& \cosh y \frac{\mathrm{d} z}{\mathrm{~d} y_{F}}-\sinh y \frac{\mathrm{d} t}{\mathrm{~d} y_{F}} \\
& =\frac{1}{T} c^{2} \frac{\partial}{\partial \theta}\left(\chi+\frac{\partial \chi}{\partial \theta}\right) \cosh \left(y-y_{F}\right)-\frac{1}{T} \frac{\partial}{\partial y_{F}}\left(\chi+\frac{\partial \chi}{\partial \theta}\right) \sinh \left(y-y_{F}\right) .
\end{aligned}
$$

Along with the expansions of hadronic matter, its temperature continues becoming lower. According to the prescription of Cooper-Frye [26], as the temperature drops to the so called chemical freeze-out temperature $T_{\mathrm{FO}}$, the inelastic collisions 
among hadrons cease. The yields of identified hadrons maintain unchanged becoming the measured results in experiments. Convoluting Equation (15) with the contributions from thermal motion, the invariant multiplicity distributions of charged particles equal [26] [27] [28]

$$
\frac{\mathrm{d}^{2} N}{2 \pi p_{T} \mathrm{~d} y \mathrm{~d} p_{T}}=\left.\frac{1}{(2 \pi)^{3}} \int \frac{\mathrm{d} N}{\mathrm{~d} y_{F}} \frac{m_{T} \cosh \left(y-y_{F}\right)}{\exp \left\{\left[m_{T} \cosh \left(y-y_{F}\right)-\mu_{\mathrm{B}}\right] / T\right\}+\delta}\right|_{T=T_{\mathrm{FO}}} \mathrm{d} y_{F},
$$

where $m_{T}=\sqrt{m^{2}+p_{T}^{2}}$ is the transverse mass of produced charged particle with rest mass $m . \mu_{\mathrm{B}}$ in Equation (17) is the baryochemical potential. For Fermi charged particles, $\delta=1$ in the denominator of Equation (17), and for Bosons, $\delta=-1$. The meaning of Equation (17) is evident. It is the convolution of $\mathrm{d} N / \mathrm{d} y_{F}$ with the energy of the charged particles in the state with temperature $T$. The former comes from the longitudinal collective flow. The latter stems from the thermal motion.

The right hand side of Equation (17) is evaluated with $T=T_{\mathrm{FO}}$. At this moment, the fluid freezes out into the charged particles. Replacing $\chi$ in Equation (16) by $\chi_{h}$ of Equation (12), it turns into

$$
\begin{aligned}
& \left.\left(\cosh y \frac{\mathrm{d} z}{\mathrm{~d} y_{F}}-\sinh y \frac{\mathrm{d} t}{\mathrm{~d} y_{F}}\right)\right|_{T=T_{\mathrm{FO}}} \\
& =\frac{1}{T_{\mathrm{FO}}}\left(\beta_{h} c_{h}\right)^{2} B\left(\theta_{\mathrm{FO}}\right)\left[S\left(\theta_{\mathrm{FO}}, y_{F}\right) \sinh \left(y-y_{F}\right)+C\left(\theta_{\mathrm{FO}}, y_{F}\right) \cosh \left(y-y_{F}\right)\right],
\end{aligned}
$$

where

$$
\begin{gathered}
S\left(\theta_{\mathrm{FO}}, y_{F}\right)=\frac{\beta_{h} y_{F}}{\lambda\left(\theta_{\mathrm{FO}}, y_{F}\right)}\left\{\frac{\beta_{h} c_{h} y_{h}\left(\theta_{\mathrm{FO}}\right)}{\lambda\left(\theta_{\mathrm{FO}}, y_{F}\right)} I_{0}\left[\lambda\left(\theta_{\mathrm{FO}}, y_{F}\right)\right]\right. \\
\left.+\left[\frac{\beta_{h}+1}{\beta_{h}}-\frac{2 \beta_{h} c_{h} y_{h}\left(\theta_{\mathrm{FO}}\right)}{\lambda^{2}\left(\theta_{\mathrm{FO}}, y_{F}\right)}\right] I_{1}\left[\lambda\left(\theta_{\mathrm{FO}}, y_{F}\right)\right]\right\}, \\
C\left(\theta_{\mathrm{FO}}, y_{F}\right)= \\
\left\{\frac{\beta_{h}+1}{\beta_{h}}+\frac{\left[\beta_{h} c_{h} y_{h}\left(\theta_{\mathrm{FO}}\right)\right]^{2}}{\lambda^{2}\left(\theta_{\mathrm{FO}}, y_{F}\right)}\right\} I_{0}\left[\lambda\left(\theta_{\mathrm{FO}}, y_{F}\right)\right] \\
+\frac{1}{\lambda\left(\theta_{\mathrm{FO}}, y_{F}\right)}\left\{\frac{y_{h}\left(\theta_{\mathrm{FO}}\right)}{c_{h}}+1-\frac{2\left[\beta_{h} c_{h} y_{h}\left(\theta_{\mathrm{FO}}\right)\right]^{2}}{\lambda^{2}\left(\theta_{\mathrm{FO}}, y_{F}\right)}\right\} I_{1}\left[\lambda\left(\theta_{\mathrm{FO}}, y_{F}\right)\right],
\end{gathered}
$$

where $I_{1}$ is the 1st order modified Bessel function of the first kind.

The integral interval of $y_{F}$ in Equation (17) is $\left[-y_{h}, y_{h}\right]$. By using Equations (15), (17), and (18)-(20), together with the definitions in Equation (13), we can get the thermal motion induced transverse momentum distributions of identified charged particles as shown Figure 1. This figure shows the transverse momentum distributions of $\pi^{ \pm}, K^{ \pm}$and $p(\bar{p})$ at mid-rapidity of $|y|<1$ in $p-p$ collisions at $\sqrt{s}=0.9,2.76$ and $7 \mathrm{TeV}$, respectively. The solid dots are the experimental data [3]. The solid curves are the results of Equation (17). The left and right columns are for the positive and negative charged particles, respectively. 


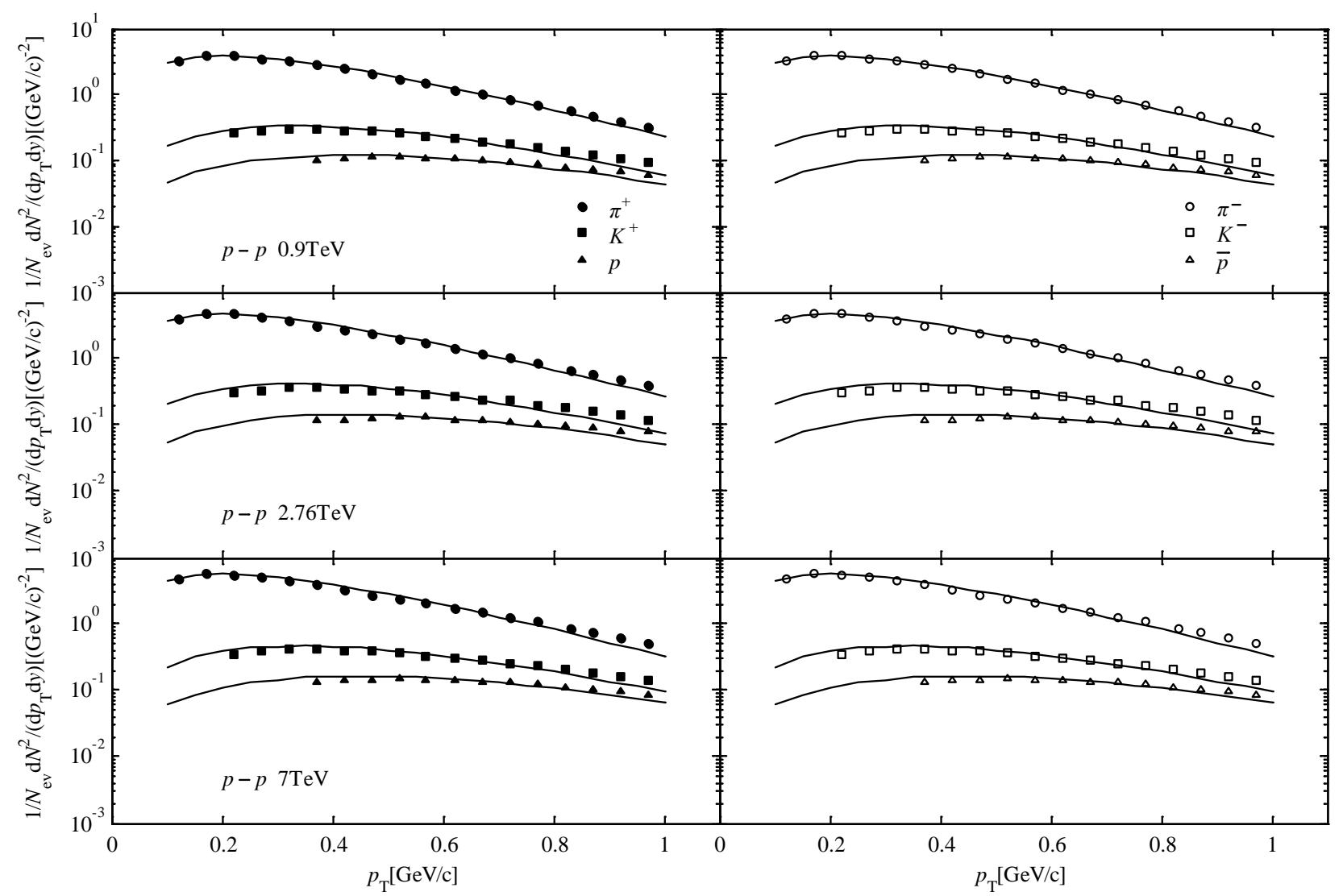

Figure 1. The transverse momentum spectra of identified charged particles produced at mid-rapidity of $|y|<1$ in $p$ - $p$ collisions at $\sqrt{s}=0.9,2.76$ and $7 \mathrm{TeV}$ (from top to bottom). The solid dots are the experimental data [3]. The solid curves are the results of Equation (17). The left and right columns are for the positive and negative charged particles, respectively.

It can be seen that the theoretical results are in good accordance with experimental data for transverse momentum up to about $p_{T}=1.0 \mathrm{GeV} / c$.

In calculations, $T_{c}$ in Equation (13) takes the well-recognized value of $T_{c}=180 \mathrm{MeV} . c_{h}$ in Equation (13) takes the value of $c_{h}=0.35$ from the investigations of Refs. [28] [48] [49] [50]. Referring to the measurements of Ref. [4], the chemical freeze-out temperature $T_{\mathrm{FO}}$ takes the value of $160 \mathrm{MeV}$ for $\pi$ in the three different incident energies of 0.9, 2.76 and $7 \mathrm{TeV}$. For $K$ and $p$, $T_{\mathrm{FO}}$ takes 170 and $175 \mathrm{MeV}$, respectively, in the three cases of collisions. The baryochemical potential $\mu_{B}$ in Equation (17) takes the value of $\mu_{B}=0$ since in the considered collisions, the abundances of particle and antiparticle are approximately equal to each other [3]. This can be clearly seen in Figure 1. As for the initial temperature $T_{0}$ in Equation (13), there is no widely accepted value so far. In this paper, consulting the results presented in Ref. [28], $T_{0}$ takes the values of 3.0, 6.5 and $7.8 \mathrm{GeV}$ for incident energies equaling $0.9,2.76$ and $7 \mathrm{TeV}$, respectively.

\section{Conclusions}

In order to see the importance of thermal motion in the expansions of the matter 
produced in $p-p$ collisions, the minor transverse collective flow is as usual ignored. The movement of matter in the transverse directions is therefore only induced by the thermal motion. Convoluted it with longitudinal collective movement, we can get the invariant multiplicity distributions of charged particles.

The longitudinal collective flow follows the relativistic hydrodynamics. The most striking feature of the model used in this paper is that it takes the phase transition into consideration, which might have appeared in the current experiments in nuclear or even in hadronic collisions. Hence, the adopted model might reflect a more realistic process of spatiotemporal evolution of matter created in collisions.

The model contains a rich information about the transport coefficients of fluid, such as the sound speed in QGP $c_{0}$, the sound speed in hadronic state $c_{h}$, the phase transition temperature $T_{c}$, the chemical freeze-out temperature $T_{\mathrm{FO}}$, the baryochemical potential $\mu_{B}$ and the initial temperature $T_{0}$. Exception for $T_{0}$, the other five coefficients take the values either from the well-known theoretical results or from experimental measurements. As for $T_{0}$, there is no much knowledge about it at present. In our calculations, $T_{0}$ is estimated referring to other researches.

The present investigations show that the $p_{T}$ distributions of charged particles measured in $p$ - $p$ collisions at LHC energies of $\sqrt{s}=0.9,2.76$ and $7 \mathrm{TeV}$ can be well described by the theoretical model for $p_{T}$ up to about $p_{T}=1.0 \mathrm{GeV} / c$. Certainly, the transverse collective flow objectively exists even though it is minor. It should affect the motion of the matter. The existing investigations have shown that if the transverse collective flow is taken into account, the describable region is extended to about $p_{T}<2.0 \mathrm{GeV} / c$ [5] [6] [7]. Furthermore, to describe the experimental data in the region of $p_{T} \geq 2.0 \mathrm{GeV} / c$, some other mechanisms, such as the method of blast-wave and perturbative QCD [8], should be introduced. Considering that the overwhelming majority of charged particles situates in the region of $p_{T}<1.0 \mathrm{GeV} / c$, we may conclude that the thermal motion plays a non-ignorable role in the transverse expansions of the matter produced in $p-p$ collisions at LHC energies.

\section{Acknowledgements}

This work is supported by the Shanghai Key Lab of Modern Optical System.

\section{References}

[1] Adamczyk, L. and STAR Collaboration (2016) Centrality Dependence of Identified Particle Elliptic Flow in Relativistic Heavy Ion Collisions at $\sqrt{\mathrm{s}_{N N}}=7.7-62.4 \mathrm{GeV}$. Physical Review C, 93, Article ID: 014907. https://doi.org/10.1103/PhysRevC.93.014907

[2] Adam, J. and ALICE Collaboration (2016) Anisotropic Flow of Charged Particles in $\mathrm{Pb}-\mathrm{Pb}$ Collisions at $\sqrt{\mathrm{s}_{N N}}=5.02 \mathrm{TeV}$. Physical Review Letters, 116, Article ID: 132302 . https://doi.org/10.1103/PhysRevLett.116.132302 
[3] Chatrchyan, S. and CMS Collaboration (2012) Study of the Inclusive Production of Charged Pions, Kaons, and Protons in pp Collisions at $\sqrt{\mathrm{s}_{N N}}=0.9,2.76$, and 7 TeV. The European Physical Journal C, 72, Article ID: 2164. https://doi.org/10.1140/epjc/s10052-012-2164-1

[4] Abelev, B.I. and STAR Collaboration (2009) Systematic Measurements of Identified Particle Spectra in pp, $\mathrm{d}+\mathrm{Au}$, and Au+Au Collisions at the STAR Detector. Physical Review C, 79, Article ID: 034909. https://doi.org/10.1103/PhysRevC.79.034909

[5] Adcox, K. and PHENIX Collaboration (2004) Single Identified Hadron Spectra from $\sqrt{\mathrm{s}_{N N}}=130 \mathrm{GeV}$ Au+Au Collisions. Physical Review C, 69, Article ID: 024904. https://doi.org/10.1103/PhysRevC.69.024904

[6] Adler, C. and STAR Collaboration (2001) Measurement of Inclusive Antiprotons from $\mathrm{Au}+\mathrm{Au}$ Collisions at $\sqrt{\mathrm{s}_{N N}}=130 \mathrm{GeV}$. Physical Review Letters, 87, Article ID: 262302. https://doi.org/10.1103/PhysRevLett.87.262302

[7] Adcox, K. and PHENIX Collaboration (2002) Centrality Dependence of $\pi^{+/-}$, $K^{+-}, p$, and $\bar{p}$ Production from $\sqrt{\mathrm{s}_{N N}}=130 \mathrm{GeV} \mathrm{Au}+\mathrm{Au}$ Collisions at RHIC. Physical Review Letters, 88, Article ID: 242301. https://doi.org/10.1103/PhysRevLett.88.242301

[8] Abelev, B. and ALICE Collaboration (2013) Centrality Dependence of $\pi, K$, and $p$ Production in $\mathrm{Pb}-\mathrm{Pb}$ Collisions at $\sqrt{\mathrm{s}_{N N}}=2.76 \mathrm{TeV}$. Physical Review $C, \mathbf{8 8}$, Article ID: 044910. https://doi.org/10.1103/PhysRevC.88.044910

[9] Alver, B and PHOBOS Collaboration (2011) Charged-Particle Multiplicity and Pseudorapidity Distributions Measured with the PHOBOS detector in $\mathrm{Au}+\mathrm{Au}$, $\mathrm{Cu}+\mathrm{Cu}, \mathrm{d}+\mathrm{Au}$, and $\mathrm{p}+\mathrm{p}$ Collisions at Ultrarelativistic Energies. Physical Review $C$, 83, Article ID: 024913. https://doi.org/10.1103/PhysRevC.83.024913

[10] Abbas, E. and ALICE Collaboration (2013) Centrality Dependence of the Pseudorapidity Density Distribution for Charged Particles in $\mathrm{Pb}-\mathrm{Pb}$ Collisions at $\sqrt{\mathrm{s}_{N N}}=$ 2.76 TeV. Physics Letters B, 726, 610-622. https://doi.org/10.1016/j.physletb.2013.09.022

[11] Adcox, K. and PHENIX Collaboration (2001) Measurement of the Midrapidity Transverse Energy Distribution from $\sqrt{\mathrm{s}_{N N}}=130 \mathrm{GeV} \mathrm{Au}+\mathrm{Au}$ Collisions at RHIC. Physical Review Letters, 87, Article ID: 052301. https://doi.org/10.1103/PhysRevLett.87.052301

[12] Arsene, I. and BRAHMS Collaboration (2005) Quark-Gluon Plasma and Color Glass Condensate at RHIC? The Perspective from the BRAHMS Experiment. Nuclear Physics A, 757, 1-27. https://doi.org/10.1016/j.nuclphysa.2005.02.130

[13] Back, B.B. and PHOBOS Collaboration (2005) The PHOBOS Perspective on Discoveries at RHIC. Nuclear Physics A, 757, 28-101.

https://doi.org/10.1016/j.nuclphysa.2005.03.084

[14] Adams, J. and STAR Collaboration (2005) Experimental and Theoretical Challenges in the Search for the Quark-Gluon Plasma: The STAR Collaboration's Critical Assessment of the Evidence from RHIC Collisions. Nuclear Physics A, 757, 102-183. https://doi.org/10.1016/j.nuclphysa.2005.03.085

[15] Adcox, K. and PHENIX Collaboration (2005) Formation of Dense Partonic matter in Relativistic Nucleus-Nucleus Collisions at RHIC: Experimental Evaluation by the PHENIX Collaboration. Nuclear Physics A, 757, 184-283.

https://doi.org/10.1016/j.nuclphysa.2005.03.086 
[16] Albrow, M.G. and British-French-Scandinavian Collaboration (1978) Studies of Proton-Proton Collisions at the CERN ISR with an Identified Charged Hadron of High Transverse Momentum at $90^{\circ}$ : (II) On the Distribution of Charged Particles in the Central Region. Nuclear Physics B, 145, 305-348. https://doi.org/10.1016/0550-3213(78)90088-3

[17] Fowler, G.N., Friedlander, E.M., Weiner, R.M. and Wilk, G. (1986) Possible Manifestation of Quark-Gluon Plasma in Multiplicity Distributions from High-Energy Reactions. Physical Review Letters, 57, 2119-2122. https://doi.org/10.1103/PhysRevLett.57.2119

[18] Friedlander, E.M. and Weiner, R.M. (1979) Evidence from Very Large Transverse Momenta of a Change with Temperature of Velocity of Sound in Hadronic Matter. Physical Review Letters, 43, 15-18. https://doi.org/10.1103/PhysRevLett.43.15

[19] Lévai, P. and Müller, B. (1991) Transverse Baryon Flow as Possible Evidence for a Quark-Gluon-Plasma Phase. Physical Review Letters, 67, 1519-1522. https://doi.org/10.1103/PhysRevLett.67.1519

[20] Alexopoulos, T. and E735 Collaboration (1993) Mass-Identified Particle Production in Proton Antiproton Collisions at $\sqrt{\mathrm{s}_{N N}}=300,540,1000$, and $1800 \mathrm{GeV}$. Physical Review D, 48, 984-997. https://doi.org/10.1103/PhysRevD.48.984

[21] Landau, L.D. (1953) On the Multiple Production of Particles in Fast Particle Collisions. Izvestiya Akademii Nauk SSSR, 17, 51-64. (In Russian)

[22] Khalatnikov, I.M. (1954) Some Questions of the Relativistic Hydrodynamics. Journal of Experimental and Theoretical Physics, 27, 529-541. (In Russian)

[23] Belen'kji, S.Z. and Landau, L.D. (1956) Hydrodynamic Theory of Multiple Production of Particles. Il Nuovo Cimento, 3, 15-31. https://doi.org/10.1007/BF02745507

[24] Hwa, R.C. (1974) Statistical Description of Hadron Constituents as a Basis for the Fluid Model of High-Energy Collisions. Physical Review D, 10, 2260-2268. https://doi.org/10.1103/PhysRevD.10.2260

[25] Bjorken, J.D. (1983) Highly Relativistic Nucleus-Nucleus Collisions: The Central Rapidity Region. Physical Review D, 27, 140-151. https://doi.org/10.1103/PhysRevD.27.140

[26] Cooper, F. and Frye, G. (1975) Landau's Hydrodynamic Model of Particle Production and Electron-Positron Annihilation into Hadrons. Physical Review D, 11, 192-213. https://doi.org/10.1103/PhysRevD.11.192

[27] Suzuki, N. (2010) One-Dimensional Hydrodynamical Model Including Phase Transition. Physical Review C, 81, 44911. https://doi.org/10.1103/PhysRevC.81.044911

[28] Mizoguchi, T., Miyazawa, H. and Biyajima, M. (2009) A Potential Including the Heaviside Function in the $1+1$ Dimensional Hydrodynamics by Landau: Its Basic Properties and Application to Data at RHIC Energies. The European Physical Journal $A, 40,99-108$. https://doi.org/10.1140/epja/i2008-10753-8

[29] Bialas, A. and Peschanski, R. (2011) Asymmetric (1 + 1)-Dimensional Hydrodynamics in High-Energy Collisions. Physical Review C, 83, Article ID: 054905. https://doi.org/10.1103/PhysRevC.83.054905

[30] Bialas, A., Janik, R.A. and Peschanski, R. (2007) Unified Description of Bjorken and Landau $1+1$ Hydrodynamics. Physical Review C, 76, Article ID: 054901. https://doi.org/10.1103/PhysRevC.76.054901

[31] Beuf, G., Peschanski, R. and Saridakis, E.N. (2008) Entropy Flow of a Perfect Fluid in $(1+1)$ Hydrodynamics. Physical Review $C$, 78, Article ID: 064909.

https://doi.org/10.1103/PhysRevC.78.064909 
[32] Wong, C.Y. (2008) Landau Hydrodynamics Reexamined. Physical Review C, 78, Article ID: 054902. https://doi.org/10.1103/PhysRevC.78.054902

[33] Csörgö, T., Nagy, M.I. and Csanád, M. (2008) New Family of Simple Solutions of Relativistic Perfect Fluid Hydrodynamics. Physics Letters B, 663, 306-311. https://doi.org/10.1016/j.physletb.2008.04.038

[34] Csanád, M., Nagy, M.I. and Lökös, S. (2012) Exact Solutions of Relativistic Perfect Fluid Hydrodynamics for a QCD Equation of State. The European Physical Journal $A$, 48, 173-178. https://doi.org/10.1140/epja/i2012-12173-7

[35] Nagy, M.I. and Csörgö, T. (2016) Simple Solutions of Fireball Hydrodynamics for Rotating and Expanding Triaxial Ellipsoids and Final State Observables. Physical Review C, 94, Article ID: 064906. https://doi.org/10.1103/PhysRevC.94.064906

[36] Sarkisyan, E.K.G., Mishra, A.N., Sahoo, R. and Sakharov, A.S. (2016) Multihadron Production Dynamics Exploring the Energy Balance in Hadronic and Nuclear Collisions. Physical Review D, 93, Article ID: 054046.

[37] Sarkisyan, E.K.G., Mishra, A.N., Sahoo, R. and Sakharov, A.S. (2016) Centrality Dependence of Midrapidity Density from $\mathrm{GeV}$ to $\mathrm{TeV}$ Heavy-Ion Collisions in the Effective-Energy Universality Picture of Hadroproduction. Physical Review D, 94, Article ID: 011501. https://doi.org/10.1103/PhysRevD.94.011501

[38] Gale, C., Jeon, S. and Schenke, B. (2013) Hydrodynamic Modeling of Heavy-Ion Collisions. International Journal of Modern Physics A, 28, Article ID: 1340011. https://doi.org/10.1142/S0217751X13400113

[39] Heinz, U. and Snellings, R. (2013) Collective Flow and Viscosity in Relativistic Heavy-Ion Collisions. Annual Review of Nuclear and Particle Science, 63, 123-151. https://doi.org/10.1146/annurev-nucl-102212-170540

[40] Sarkisyan, E.K.G. and Sakharov, A.S. (2010) Relating Multihadron Production in Hadronic and Nuclear Collisions. The European Physical Journal C, 70, 533-541. https://doi.org/10.1140/epjc/s10052-010-1493-1

[41] Mishra, A.N., Sahoo, R., Sarkisyan, E.K.G. and Sakharov, A.S. (2014) Effective-Energy Budget in Multiparticle Production in Nuclear Collisions. The European Physical Journal C, 74, 3147. https://doi.org/10.1140/epjc/s10052-014-3147-1

[42] Wang, Z.W., Jiang, Z.J. and Zhang, Y.S. (2009) Charged-Particle Pseudorapidity Distributions in $\mathrm{Au}+\mathrm{Au}$ Collisions at High Energies. Journal of University of Shanghai for Science and Technology, 31, 322-326. (In Chinese)

[43] Jiang, Z.J., Li, Q.G. and Zhang, H.L. (2013) Revised Landau Hydrodynamic Model and the Pseudorapidity Distributions of Charged Particles Produced in Nucleus-Nucleus Collisions at Maximum Energy at the BNL Relativistic Heavy Ion Collider. Physical Review C, 87, Article ID: 044902. https://doi.org/10.1103/PhysRevC.87.044902

[44] Jiang, Z.J., Zhang, Y., Zhang, H.L. and Deng, H.P. (2015) A Description of the Pseudorapidity Distributions in Heavy Ion Collisions at RHIC and LHC Energies. Nuclear Physics A, 941, 188-200. https://doi.org/10.1016/j.nuclphysa.2015.06.015

[45] Noronha-Hostler, J., Luzum, M. and Ollitrault, J.-Y. (2016) Hydrodynamic Predictions for 5.02 TeV Pb-Pb Collisions. Physical Review C, 93, Article ID: 034912. https://doi.org/10.1103/PhysRevC.93.034912

[46] Martinez, M., McNelis, M. and Heinz, U. (2017) Anisotropic Fluid Dynamics for Gubser Flow. Physical Review C, 95, Article ID: 054907. https://doi.org/10.1103/PhysRevC.95.054907

[47] Jiang, Z.J., Hui, J.Q. and Zhang, Y. (2017) The Rapidity Distributions and the 
Thermalization Induced Transverse Momentum Distributions in Au-Au Collisions at RHIC Energies. Advances in High Energy Physics, 2017, Article ID: 6896524. https://doi.org/10.1155/2017/6896524

[48] Adare, A. and PHENIX Collaboration (2007) Scaling Properties of Azimuthal Anisotropy in $\mathrm{Au}+\mathrm{Au}$ and $\mathrm{Cu}+\mathrm{Cu}$ Collisions at $\sqrt{\mathrm{s}_{N N}}=200 \mathrm{GeV}$. Physical Review Letters, 98, Article ID: 162301. https://doi.org/10.1103/PhysRevLett.98.162301

[49] Gao, L.N., Chen, Y.H., Wei, H.R. and Liu, F.H. (2014) Speed of Sound Parameter from RHIC and LHC Heavy-Ion Data. Advances in High Energy Physics, 2014, Article ID: 450247.

[50] Borsányi, S., Endrődi, G., Fodor, Z., Jakovác, A., Katz, S.D., Krieg, S., Ratti, C. and Szabó, K.K. (2010) The QCD Equation of State with Dynamical Quarks. Journal of High Energy Physics, 77, 1-31. https://doi.org/10.1007/JHEP11(2010)077 